\title{
A Comparative Study of Hedges Use Between American and Chinese Students’ Writing
}

\author{
Harrison Geng-han Lu, Chelsea Chen-xu Fu \\ Northern Arizona University, Flagstaff, Arizona, USA
}

\begin{abstract}
In college-level academic writings, the appropriate use of hedges is an important skill to mark a writer's stances pragmatically. This skill is not easy for English as Foreign Language (EFL) students to acquire, especially for Chinese students whose first language (L1) is dramatically different from English. This study compared writings by Chinese students and American students to pinpoint the similarities and differences between the two groups in their uses of various hedging devices. A set of first-year writings by the two groups was collected at Northern Arizona University (NAU). In their writings, five types of hedges were investigated respectively, namely model auxiliary type, lexical verb type, adjective/adverb type, phrase type, and sentence structure type. The results show that Chinese students use as many hedges as American students in general, but the two populations differ from each other in their use of lexical verbs in particular.
\end{abstract}

Keywords: Chinese students, American students, academic writing, hedges

\section{Introduction}

The term "hedge" refers to lexical and syntactic features modifying a writer's or a speaker's stance (Hinkel, 1996). In academic writing, the appropriate use of hedges is an important skill to moderate the writers' own claims and to show uncertainty when addressing other people's works. This skill, however, is not easy for English as Second Language (ESL) and English as Foreign Language (EFL) students to acquire, especially for Chinese students whose first language (L1) is dramatically different from English. Compared with native English speakers, Chinese students might overuse, or rarely use this strategy.

This research examined the frequencies of different types of hedges employed by Chinese students and American students in their first-year college-level academic writings. This comparative study has pedagogical implications on teaching English academic writing to Chinese ESL/EFL students. On one hand, this study potentially benefits ESL/EFL teachers when they teach Chinese students academic writing skills. For example, Chinese students rarely use lexical verb type of hedges in their writings; the teachers could focus more on this type. On the other hand, the use of hedges is common in various registers of formal writing, such as writing emails to professors, scientific reports, and so on. This study could help the students raise their awareness of this skill and improve their writings in different registers.

Apart from the practical benefits, this comparative study also has theoretical implications on the research of hedges. This study associates different types of hedges with students' writings from a cross-culture

Harrison Geng-han Lu, MA TESL candidate, English Department, Northern Arizona University.

Chelsea Chen-xu Fu, MA TESL candidate, English Department, Northern Arizona University. 
perspective. Through the comparison, how Chinese English learners differ from native English speakers in their choices of hedges is clearly presented. Although there have been empirical studies on hedges in relation to academic writings, most of them discussed hedges in different genres of journal articles (HU \& CAO, 2011). In response to this lack of attention to students' writings, this research provides a new insight on hedges use in this less investigated genre.

Previous studies of hedges have discussed hedges from various perspectives. In terms of its discourse function, Hyland (1998) argued that hedging is the use of words and expressions whose job is to make things less fuzzy. As a linguistic device, it could qualify a speaker's confidence in the truth of a proposition. In his book, Hyland provided a comprehensive account of hedging in scientific articles from pragmatic viewpoint. He found that hedges are more frequently distributed in Results and Discussion sections in scientific articles. Also from pragmatic and discourse perspectives, Markkanen and Schröder (1997) synthesized different definitions and functions of hedges proposed by previous scholars. They concluded that the concept of hedges originated from logic and semantics, while is now extending to pragmatics and discourse fields.

Apart from the above non-empirical studies of hedges, there have been empirical and comparative studies investigating the use of hedges in second language (L2) writings. Hinkel (1996) compared native English speakers and non-native speakers in the frequencies of various types of hedges and intensifiers in academic prose. The results showed that non-native speakers employ limited range of hedges devices compared with native English speakers. Wishnoff (2000) claimed that learning and using hedges in English writing are beneficial for L2 English students to build up a sense of inter-language pragmatics. Explicit teaching of hedges in EFL instruction could help L2 students increase their awareness of hedges use and improve their ability to use this device. HU and CAO (2011) compared the use of hedges and boosters between L1 English scholars and Chinese scholars in English-medium and Chinese-medium abstracts. Three parallel sub-corpora were constructed: (1) the CA-CJ sub-corpus (i.e., Chinese abstracts published in the Chinese journals), (2) the EA-CJ sub-corpus (i.e., English abstracts published in the Chinese journals), and (3) the EA-EJ sub-corpus (i.e., English abstracts published in the English journals). In the three sub-corpora, empirical studies and non-empirical studies were separated. HU and CAO concluded that English abstracts use hedges more frequently than Chinese abstracts; while Chinese abstracts use more boosters than the English counterparts. What is more, the abstracts of the empirical research articles use significantly more boosters than non-empirical academic articles.

Based on the discussion above, there have been very few empirical studies about the use of hedges by EFL/ESL learners. This study compared the frequencies of hedges by Chinese ESL students and American students in their first-year academic writings. Five sub-types of hedges were investigated respectively, namely model auxiliary type, lexical verb type, adjective/adverb type, phrase type, and sentence structure type. More specifically, six research questions are listed as follows:

(1) Does the frequencies of model auxiliary type of hedges (i.e., might, could, would) differ from Chinese students' writings to American students' writings?

(2) Does the frequencies of lexical verb type of hedges (i.e., seem, assume, suggest) differ from Chinese students' writings to American students' writings?

(3) Does the frequencies of adjective/adverb type of hedges (i.e., probable, possible, probably, possibly, likely, perhaps, mainly) differ from Chinese students' writings to American students' writings?

(4) Does the frequencies of phrase type of hedges (i.e., in general, to some extent, to certain extent) differ from Chinese students' writings to American students' writings? 
(5) Does the frequencies of sentence structure type of hedges (i.e., passive voice and raising structure) differ from Chinese students' writings to American students' writings?

(6) Does the frequencies of total use of hedges (i.e., all types summed up) differ from Chinese students' writings to American students' writings?

As previous studies showed, it is possible that there are differences in the frequencies of hedges between Chinese ESL students and American students. The frequencies of various types of hedges might be less in Chinese students' writings.

\section{Method}

In this section, data sources, measurements, and procedures are discussed. In data sources section, where and how the sample writings were selected are addressed; in measurements section, how research variables are measured is illustrated; in procedure section, research design, analysis of the results, and administration of the research are explained.

\section{Data Sources}

In this study, the sample writings were selected from the students' writing assignments in Critical Reading and Writing in the University Community class (ENG 105) at Northern Arizona University (NAU). Thirty comprehensive argumentation papers by American students and 30 by Chinese ESL students were collected. The reasons of choosing writing assignments from ENG 105 class are as follows. Firstly, in a comprehensive argumentation paper, the students could use hedges to either moderate or support their own ideas. What is more, the topics of their writings vary from drug issue to public transportation. While writing on those topics, the students need to cite other people's works as well to express their own opinions. This requires the use of hedges to moderate the writers' stances. Secondly, before taking this writing class, Chinese students possibly have not received instructions of hedges in high school level English class in China. Without systematic knowledge of using hedges, the students might write in different ways from native English speakers. Thirdly, even if the Chinese students at NAU learned about hedges, they might have preferences of certain types of hedges, compared with native speakers. This is because ENG 105 is an introductory course, where the use of hedges is not a main focus. Based on the above discussion, ENG 105 writing assignments are appropriate for this study.

During the process of samples selection, the researchers identified the L1 of the students by their names. As this collection of students' writings are secondary data, the students' personal information was unknown, including their L1. For American students, the researchers selected those whose last names are typical American ones, such as "Brown", "White", and so on. For Chinese students, their names are dramatically different from students from other countries. Chinese names have no middle initials and the surnames are easy to identify on the name list. In most cases, their names include two or three Chinese characters, such as 张三 (San Zhang). Zhang is a typical Chinese surname and is easy to identify. Some may question how to distinguish American born Chinese from Chinese students. In most cases, American born Chinese students have English middle names while Chinese ESL students do not. By this way, the papers written by American students and Chinese students were identified.

\section{Measures}

This research study investigated whether the students' L1 influences their choices of hedges in English academic writings. The students' L1 is the independent variable, while the frequencies of hedges use belong to 
the dependent variable, which is the focus of this study. In terms of the students' L1, this study investigated Chinese students and American English speakers in particular; the choices of hedges are classified into five types, based on the types classified by HU and CAO (2011). The five types of hedges include model auxiliary type (i.e., might, could, would), lexical verb type (i.e., seem, assume, suggest), adverbs/adjectives type (i.e., probable/probably, possible/possibly, perhaps, likely, mainly), phrase type (i.e., in general, to some/a certain extent), and sentence structures type (i.e., passive structure, it is important that...). In the process of counting frequencies of each type, two raters counted separately. According to Pearson Correlation test, the inter-rater reliability is very strong $(r=.995$, see Appendix A).

\section{Procedure}

This study is a quasi-experimental study, from the post-positivism philosophical stance. The researchers neither expected certain results or to test an existing theory. In order to be unbiased and impartial, the data were collected objectively, and the judgment on types of hedges was based on rigorous discussion of previous studies. The present study compared the frequencies of each type of hedges in Chinese EFL students' and American students' writings. It should be noted that, there was no pre-assumption that American students' choices of hedges would be appropriate all the time. In this study, whether every hedge use is appropriate or not in not included.

Analysis. This study investigated whether Chinese students and American students choose different types of hedges in their freshmen year college academic writings. The null hypothesis is that Chinese students and American students have no difference in the frequencies of each type of hedges use and the frequencies of total use of hedges. The students' L1 is a nominal independent variable, which was divided into Chinese (1 = Chinese) and American English (2 = American English). The frequency of types of hedges is an interval dependent variable. The frequencies of the choices were counted by AntConc. The numbers of hedges use are normalized to 1,000 words per essay. After the data were collected, SPSS 17.0 was used to generate the results.

Administration. In order to access the data-based student writing samples of ENG 105, the researchers contacted Director of English Composition for permission. The researchers selected 30 argumentation papers written by Chinese students and 30 argumentation papers written by American students by identifying their names on the papers. All the texts were converted into plain text documents which can be put into AntConc for analysis. The papers were stored in a password-protected computer that can only be accessed by the researchers. The names of the students were altered by identity numbers in sequential order.

\section{Results}

In this section, firstly, the descriptive statistics of mean frequencies and standard deviation are reported. Secondly, the hypothesis testing of statistical procedure, results, and practical significance are presented. In this quantitative analysis, the alpha was set at .05 level (2-tailed) for the Mann Whitney U test.

\section{Descriptive Statistics}

After the raw frequencies were obtained, the total words of each essay were normalized to 1,000 words.

Table 1 and Table 2 present the descriptive statistical information of the five types of hedges used by Chinese students and American students. The means of auxiliary type, adjective/adverb type, sentence type, and total use of hedges show no significant difference in Chinese group and American group. However, the means of lexical verb type of hedges in Chinese group (mean $=.29$ ) and American group (mean $=.87$ ) display a 
noticeable difference. Also, there is a difference between Chinese students (mean $=.23$ ) and American students (mean $=.06$ ) in the mean of phrase type hedges. In terms of standard deviation, Table 1 shows that the sentence structures type of hedge has the largest standard deviation $(S D=9.09)$, which illustrates that in the Chinese students group, some Chinese students tend to use sentence type of hedges frequently while others seldom use it. Lexical verb type has the smallest standard deviation $(S D=.48)$, showing the fact that Chinese students use less lexical verb type of hedges. According to Table 2, there is a large intra-group difference when American students use sentence type hedges $(S D=7.24)$. The smallest difference intra-group difference exists in the phrase type of hedges $(S D=.22)$. In the following part, the statistical procedures, statistical results, and the practical significance of the results are discussed.

Table 1

Descriptive Statistics of the Frequencies of Hedges by Chinese Students

\begin{tabular}{llccrrr}
\hline & $N$ & Range & Minimum & Maximum & Mean & $S D$ \\
\hline Aux_N & 30 & 29.36 & 0.00 & 29.36 & 7.8601 & 7.14798 \\
Verb_N & 30 & 1.51 & 0.00 & 1.51 & .2955 & .48186 \\
Adjv_N & 30 & 7.84 & 0.00 & 7.84 & 1.8691 & 2.09225 \\
Phrase_N & 30 & 1.68 & 0.00 & 1.68 & .2273 & .48775 \\
Sentence_N & 30 & 34.06 & 7.41 & 41.47 & 21.4076 & 9.09147 \\
Total_N & 30 & 55.82 & 12.97 & 68.79 & 31.6595 & 12.65264 \\
Valid N (listwise) & 30 & & & & &
\end{tabular}

Note. "Aux_N" denotes the normalized frequencies of model auxiliaries type; "Verb_N" denotes the normalized frequencies of lexical verbs type; “Adjv_N” denotes the normalized frequencies of adjectives/adverbs type; "Phrase_N” denotes the normalized frequencies of phrases type; "Sentence_N" denotes the normalized frequencies of sentence structures type; and "Total_N" denotes the normalized frequencies of total numbers of hedges.

Table 2

Descriptive Statistics of the Frequencies of Hedges by American Students

\begin{tabular}{llccrrr}
\hline & $N$ & Range & Minimum & Maximum & \multicolumn{1}{c}{ Mean } & \multicolumn{1}{c}{$S D$} \\
\hline Aux_N & 30 & 21.88 & 0.00 & 21.88 & 8.1793 & 5.01986 \\
Verb_N & 30 & 4.06 & 0.00 & 4.06 & .8725 & 1.04011 \\
Adjv_N & 30 & 6.36 & 0.00 & 6.36 & 1.9115 & 1.67048 \\
Phrase_N & 30 & .97 & 0.00 & .97 & .0567 & .21825 \\
Sentence_N & 30 & 26.43 & 8.28 & 34.71 & 20.6800 & 7.24334 \\
Total_N & 30 & 37.79 & 10.35 & 48.14 & 31.7000 & 10.61265 \\
Valid N (listwise) & 30 & & & & & \\
\hline
\end{tabular}

\section{Hypotheses Testing Results}

In this study, normalized frequencies of five sub-types and total hedges use are investigated. Therefore, there are six hypotheses that need to be tested, as listed from hypothesis 1 to 6 .

(1) There is no difference in the frequencies of model auxiliary type of hedges (i.e., might, could, would) between Chinese students' writings and American students' writings.

(2) There is no difference in the frequencies of lexical verb type of hedges (i.e., seem, assume, suggest) between Chinese students' writings and American students' writings.

(3) There is no difference in the frequencies of adjective/adverb type of hedges (i.e., probable, possible, probably, possibly, likely, perhaps, mainly) between Chinese students' writings and American 
students' writings.

(4) There is no difference in the frequencies of phrase type of hedges (i.e., in general, to some extent, to certain extent) between Chinese students' writings and American students' writings.

(5) There is no difference in the frequencies of sentence structure type of hedges (i.e., passive voice and raising structure) between Chinese students' writings and American students' writings.

(6) There is no difference in the frequencies of total use of hedges (i.e., all types summed up) between Chinese students' writings and American students' writings.

In the above six hypotheses, the independent variables are the students' L1s, while the dependent variables are the frequencies of total use of hedges and the frequencies of each type of hedges use. This is a between groups designed study where the frequencies of hedges use are categorized by L1. In this case, independent t-test should be used if the data are normally distributed. If not, Mann Whitney $U$ test should be chosen. According to the histogram normal curves (see Appendix B) for the five types of hedges, the data are not normally distributed. Therefore, Mann Whitney $\mathrm{U}$ was used to test the above null hypothesis. The original results are attached in Appendix C.

Table 3

Table of $z$ Values From the Five Mann Whitney U Tests

\begin{tabular}{lllllll}
\hline & Aux_N & Verb_N & Adjv_N & Phrase_N & Sentence_N & Total_N \\
\hline$z_{\text {obs }}$ & -0.991 & -2.396 & -0.541 & -1.576 & -0.089 & -0.444 \\
$z_{\text {crit }}$ & \pm 1.960 & \pm 1.960 & \pm 1.960 & \pm 1.960 & \pm 1.960 & \pm 1.960 \\
Statistical Results & $z_{\text {obs }}<z_{\text {crit }}$ & $z_{\text {obs }}>z_{\text {crit }}$ & $z_{\text {obs }}<z_{\text {crit }}$ & $z_{\text {obs }}<z_{\text {crit }}$ & $z_{\text {obs }}<z_{\text {crit }}$ & $z_{\text {obs }}<z_{\text {crit }}$ \\
\hline
\end{tabular}

Note. In the tests, $p=.05$, two-tailed distribution are used. The observed values are round up to three digits after the decimal.

In Table 3, the statistical results in column 1, 3, 4, 5, and 6 show that there is no difference between Chinese students and American students in their frequencies of hedges use among auxiliary type, adjective and adverb type, phrases type, sentence type as well as total use $\left(z_{o b s}<z_{c r i t}\right)$. However, there is a difference in the frequencies of lexical verb type between Chinese students and American students $\left(z_{o b s}>z_{c r i t}\right)$. Therefore, hypothesis 2 can be rejected. The following step calculates the strength of association-eta squared $\left(\eta^{2}\right)$. According to Hatch and Lazaraton (1990), the formula of $\eta^{2}$ for Mann Whitney $\mathrm{U}$ test is used as the following:

$$
\eta 2=\frac{z_{o b s}^{2}}{N-1}=\frac{(-2.396)^{2}}{60-1}=.097
$$

The strength of association $\left(\eta^{2}=.097\right)$ shows that although there is a difference in the frequencies of lexical verb type of hedges between Chinese students and American students, the difference is not practically significant. The following two figures demonstrate the comparison between Chinese students and American students in the frequencies of each type of hedges. Figure 1 shows the normalized frequencies in a scale from 0 to 1,000 for each type of hedges. Figure 2 highlights the lexical verb type in a scale from 0 to 30.

Generally speaking, the total frequencies of hedges are similar in both the two groups. If, observing each type respectively, there are no differences between Chinese and American students in the frequencies of model auxiliary type, adjective/adverb type, phrase type, and sentence structures type in their writings, as shown in Figure 1. However, the two groups differ in the frequencies of lexical verb type, as shown in Figure 2, the "zoom in” of lexical verbs type in Figure 1. According to Figure 2, American students used twice numbers of lexical verb hedges than the Chinese students did. 


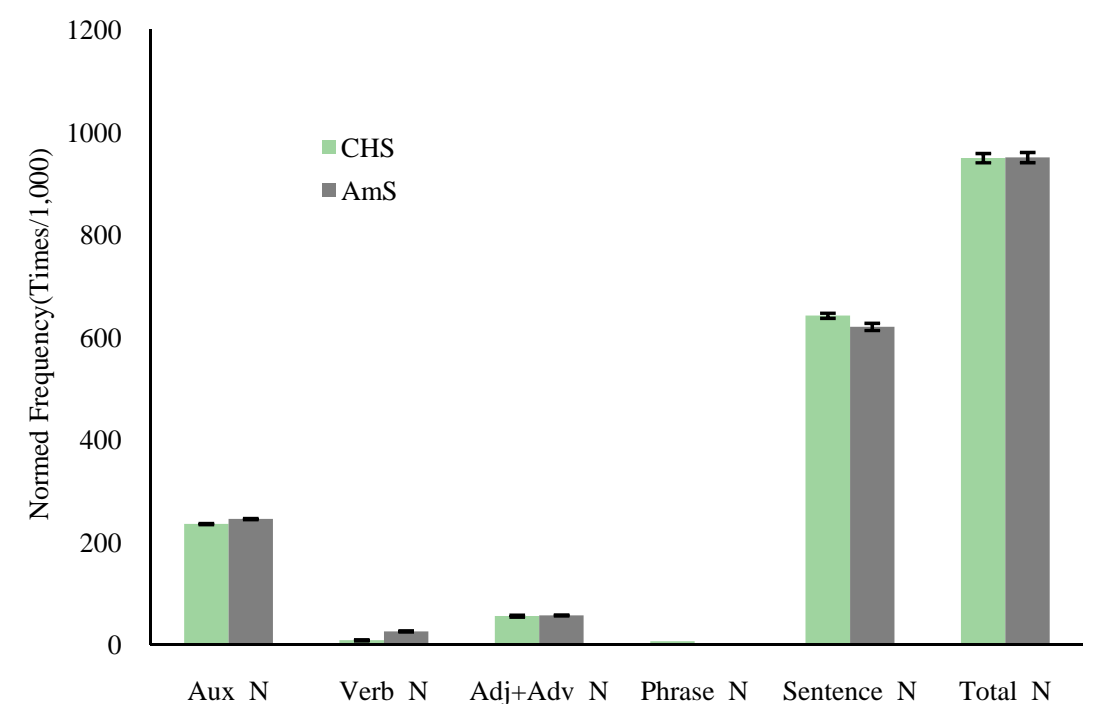

Figure 1. The frequencies of five types of hedges and total hedges.

“CHS” stands for Chinese student while “AmS” stands for American student.

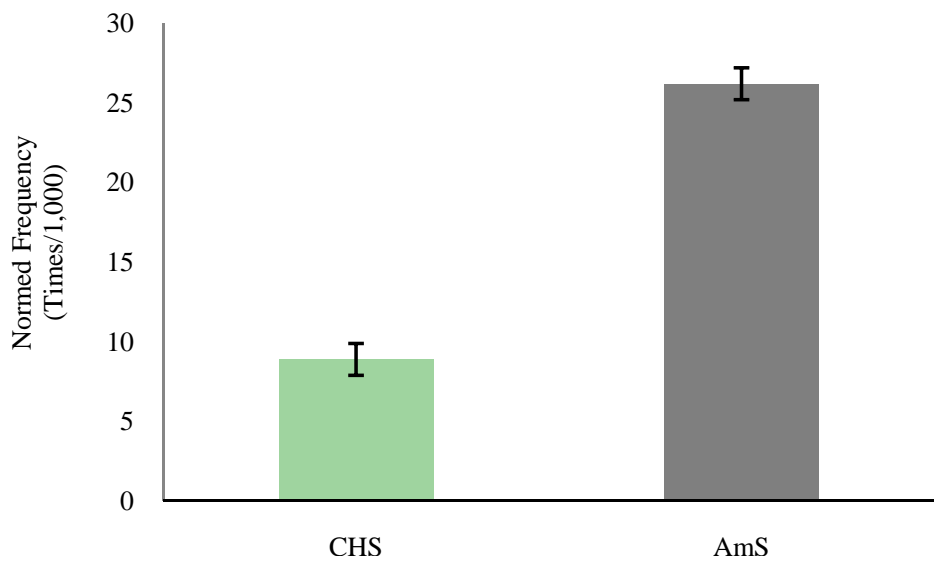

Figure 2. The frequencies of lexical verb type of hedges.

\section{Discussion}

Based on the results of statistical test, the only difference between Chinese students and American students exists in verb hedges use. The difference, however, is not practically significant. This results contrast the results in previous comparative studies by Hinkel (1996) and HU and CAO (2011). They claimed that English native speakers differ from non-native speakers in their frequencies of hedges use in academic prose. HU and CAO pointed out that the frequencies of hedges use in the abstracts written by native English speakers are more frequently than those of Chinese scholars. This study indicates that the Chinese students are aware of the use of hedges in academic writings. The only problem for them is lacking the knowledge of lexical verb type hedges. The following interpretations could explain the difference between the results in this study and those in previous literature.

Firstly, the lack of lexical verb type usage in Chinese students' writings could be accounted by literal translation. Most Chinese students took ENG 105 class in their first academic year after they arrived in the 
United States. Many of them use literal translation when writing in English. However, this strategy of writing is not appropriate because an English word does not necessarily match a corresponding Chinese word semantically. HU and CAO (2011) concluded that the lexical verb type of hedges in Chinese include juede (feel), qingxiang (tend to), and changshi (attempt), which are not in perfect accordance with English. Because of this, the Chinese students are not sensitive to the lexical verb type of hedges in English, thus they may not use them frequently as American students do.

Secondly, the writing improvement programs on campus (e.g., "university literacy center” or "university writing center”) provide lots of assistance to international students' writings. Therefore, the Chinese students could have asked English tutors to revise their writing assignments. It is possible that the English tutors gave suggestions or made the changes in the use of hedges. As a result, the total use of hedges in Chinese students' writings and American students' writing has no significant difference. With the help of the instruction in the writing improvement programs, Chinese students who do not use hedges could have received advice from native English speakers who might pay attention to the use of hedges.

This study has some limitations on the sample selection. There are some potential threats to the internal validity in this study. All the participants are first year students at NAU. The results in this study might not represent all the Chinese EFL students and American students as well. In addition, there are only 60 writing samples in the collection of this study. The small sample size could also influence the results. What is more, different Chinese students' English learning backgrounds might also affect their word choices in English writings. In terms of the threats to the external validity, different instructors of ENG 105 may give lectures in different ways. It is possible that some of them paid particular attention to the use of hedges while some might not.

The results of this study have some implications on teaching Chinese students' English writings. Though Chinese students' writings in this study used as many hedges as American students' writings, there were very few lexical verbs such as "assume”, “seem”, “suggest”, and so on. In classroom instruction, teachers could emphasize this type in particular. Further, future studies can focus on not only the frequencies, but also the accuracy of the use of hedges in ESL students' writings.

\section{Conclusion}

This study proves the fact that among the six types of hedges, there is a statistical difference in the frequencies of lexical verb type between Chinese students' writings and American students' writings. The results reveal the hedge use patterns in Chinese students' and American students' first-year writings, which could be utilized to enhance the English literacy teachers' writing instruction in the fields of ESL/EFL. The results are also helpful for first-year composition teachers to recognize international students, especially Chinese students' hedge use habits in their academic writing.

\section{References}

FENG, G. (2008). Pragmatic markers in Chinese. Journal of Pragmatics, 40, 1687-1718.

GAO, Y., LI, L., \& LU, J. (2001). Trends in research methods in applied linguistics: China and the West. English for Specific Purposes, 20, 1-14.

Hatch, E., \& Lazaraton, A. (1990). The research manual: Design and statistics for applied linguistics. Cambridge, MA: Newbury House. 
Hinkel, E. (1996). Hedging, inflating, and persuading in L2 academic writing. In Applied language learning (pp. 29-54). Monterey, CA: Presidio of Monterey, Calif.

HU, G., \& CAO, F. (2011). Hedging and boosting in abstracts of applied linguistics articles: A comparative study of English and Chinese-medium journals. Journal of Pragmatics, 43, 2795-2809.

Hyland, K. (1998). Hedging in scientific research articles. Amsterdam, Netherlands: John Benjamins Pub. Co..

LIU, L. (2005). Rhetorical education through writing instruction across cultures: A comparative analysis of select online instructional materials on argumentative writing. Journal of Second Language Writing, 14, 1-18.

Markkanen, R., \& Schröder, H. (1997). Hedging and discourse: Approaches to the analysis of a pragmatic phenomenon in academic texts. Berlin, Germany: Walter de Gruyter.

Norušis, M. J., \& SPSS Inc. (2008). SPSS statistics 17.0 guide to data analysis. Upper Saddle River, N.J: Prentice Hall.

Wishnoff, J. (2000). Hedging your bets: L2 learners' acquisition of pragmatic devices in academic writing and compute-mediated discourse. Second Language Studies, 19(1), 119-148.

Appendix A: The Pearson Correlation for Inter-rater Reliability

\begin{tabular}{llll}
\hline & & Genghan & Chenxu \\
\hline Genghan & Pearson Correlation & 1 & .995 \\
& Sig. (2-tailed) & & .000 \\
N & 60 & 60 \\
Chenxu & Pearson Correlation & .995 & 1 \\
& Sig. (2-tailed) & .000 & \\
& $\mathrm{~N}$ & 60 & 60 \\
\hline
\end{tabular}

Note. Correlation is significant at the 0.05 level (2-tailed).

Appendix B: The Histograms of Each Type of Hedges Use and Total Hedges Use

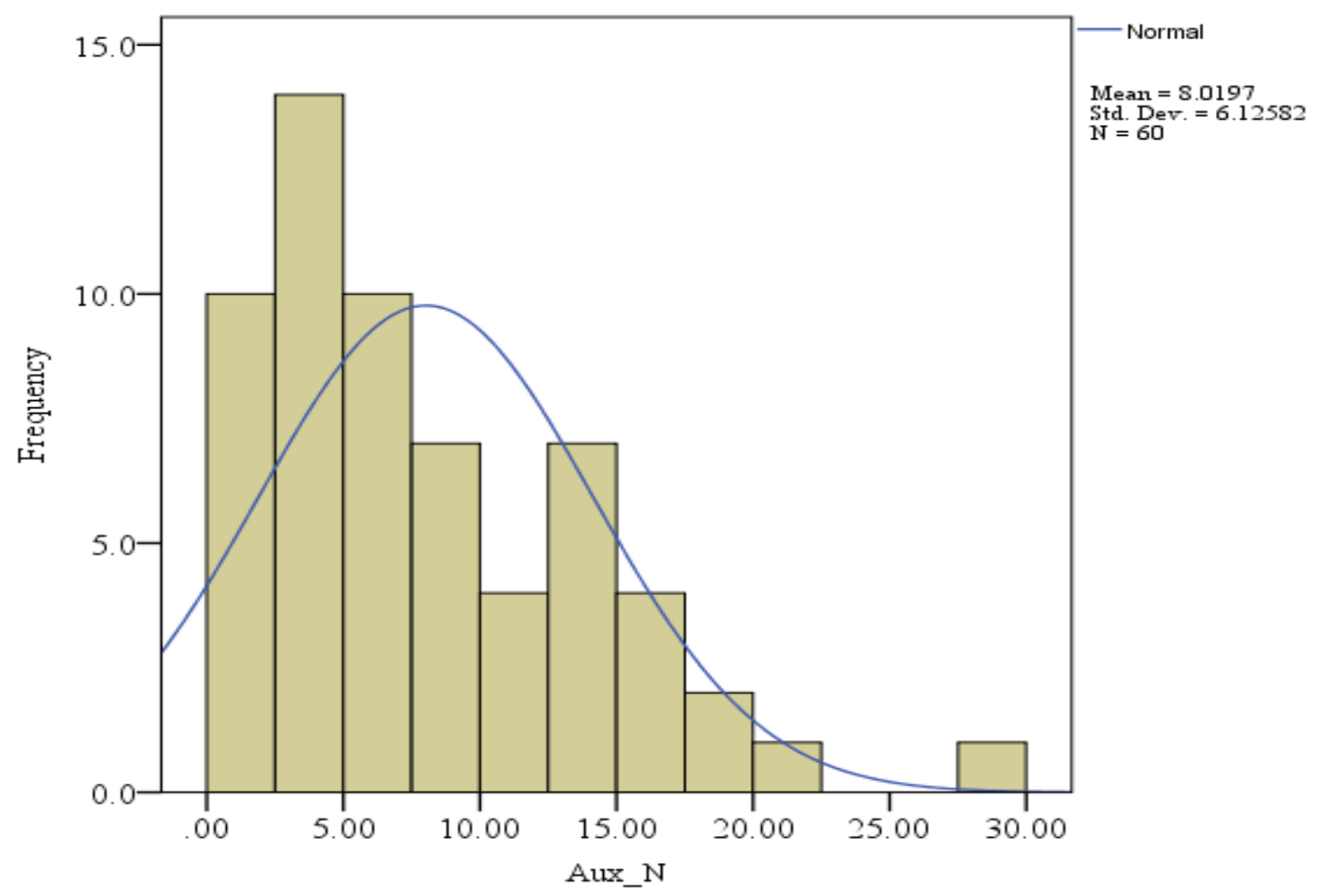

Figure B1. The histogram of frequency distribution of model auxiliary type. 


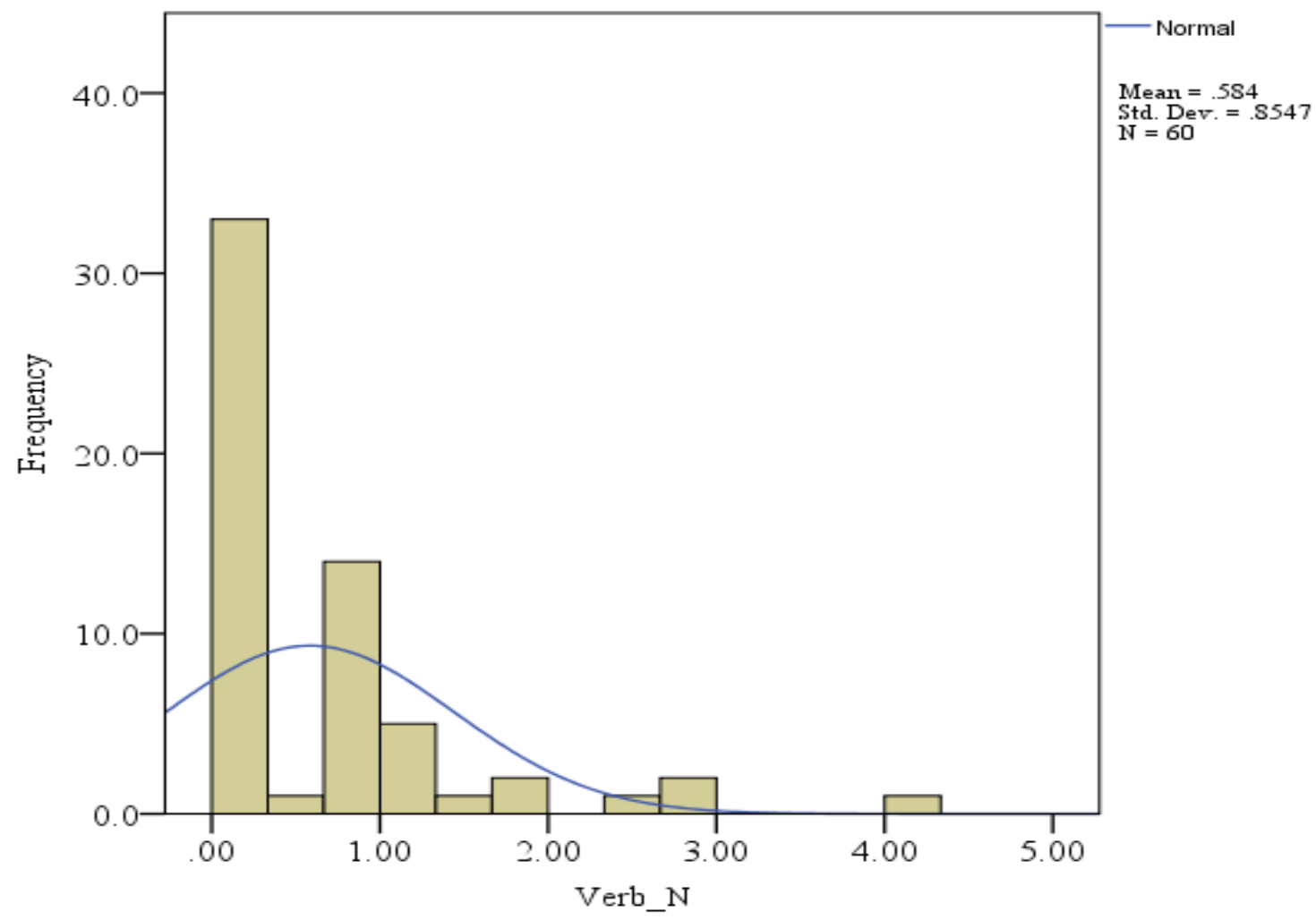

Figure B2. The histogram of frequency distribution of lexical verb type.

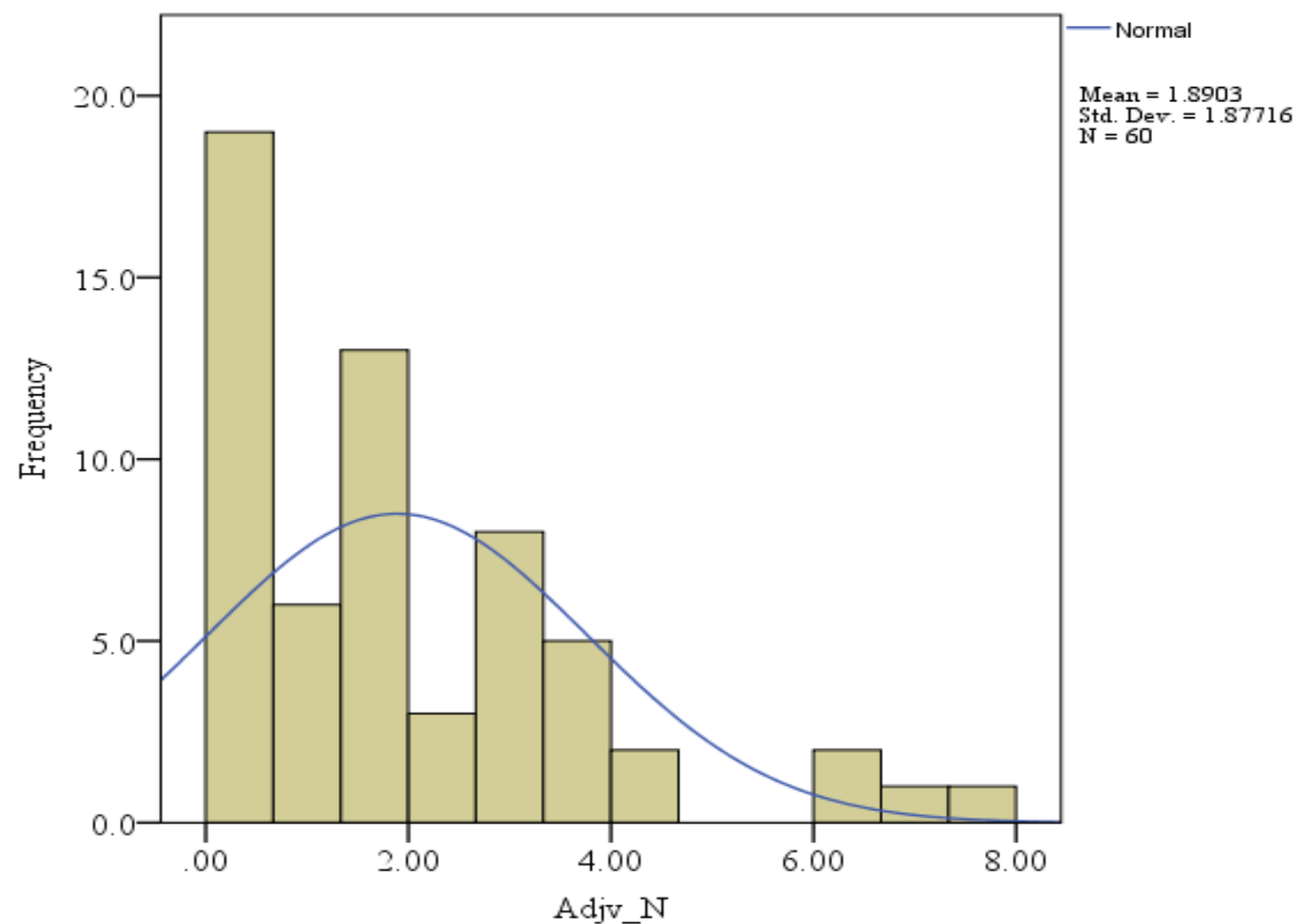

Figure B3. The histogram of frequency distribution of adjective/adverb type. 


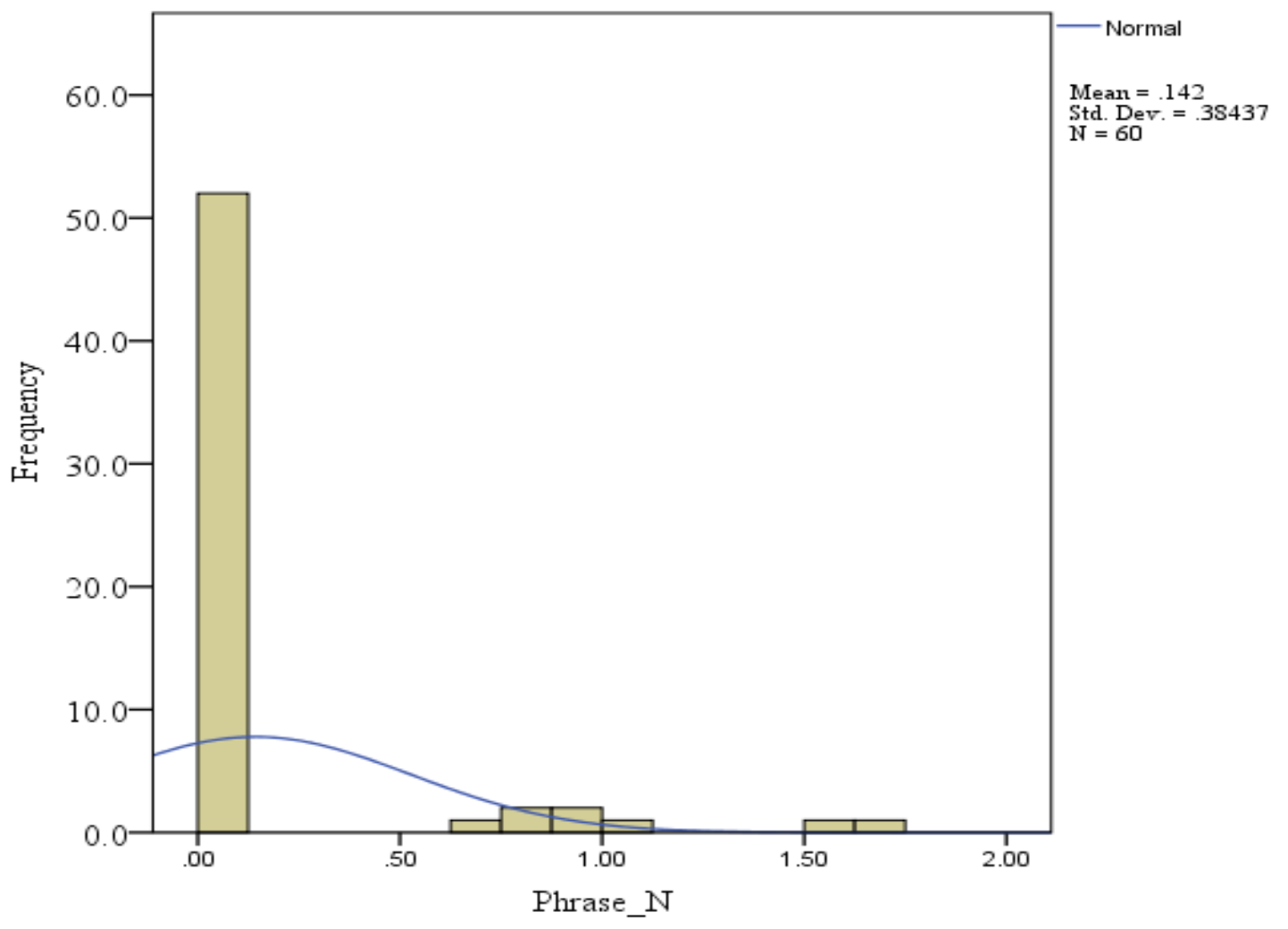

Figure B4. The histogram of frequency distribution of phrase type.

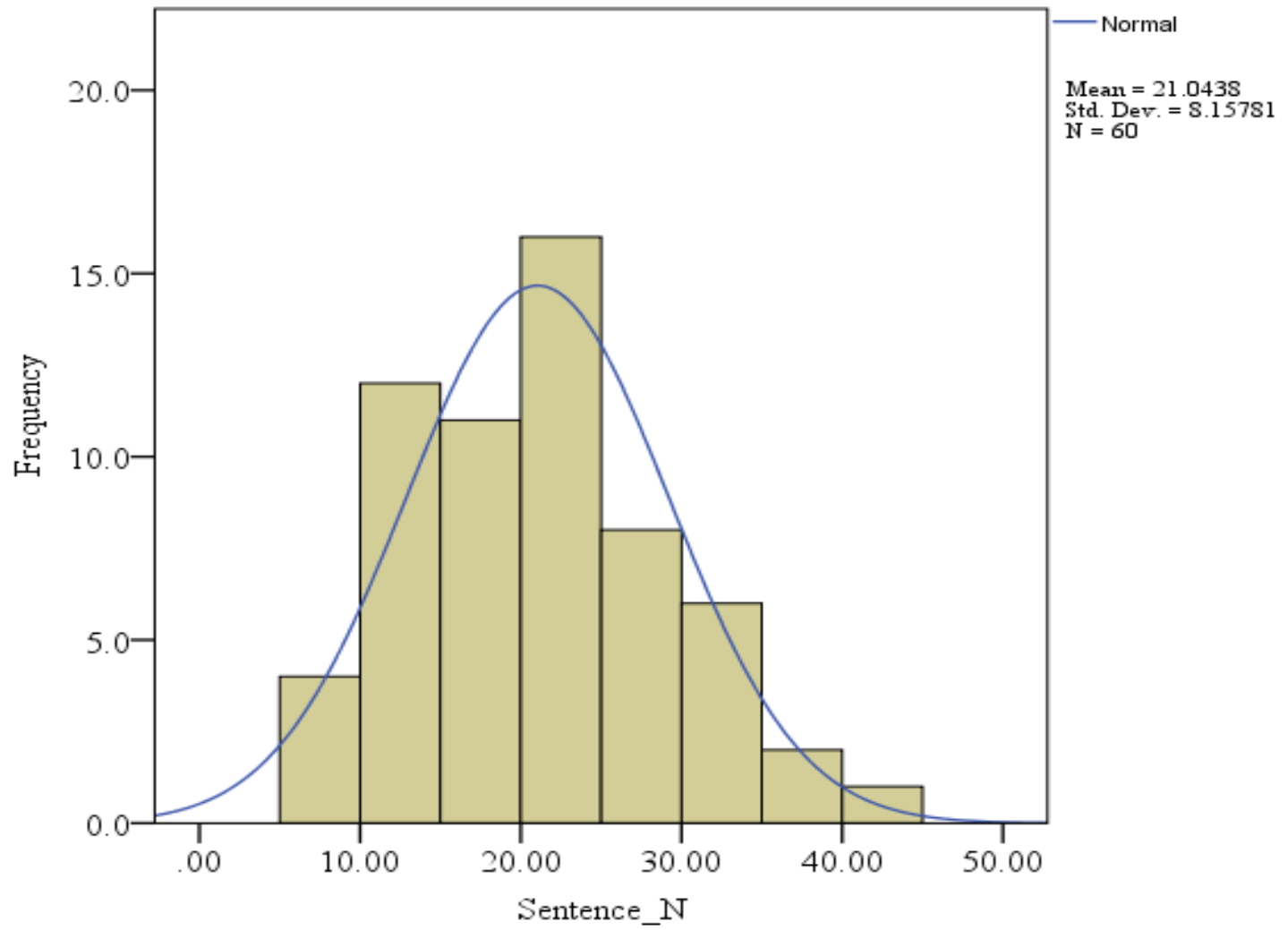

Figure B5. The histogram of frequency distribution of sentence type. 


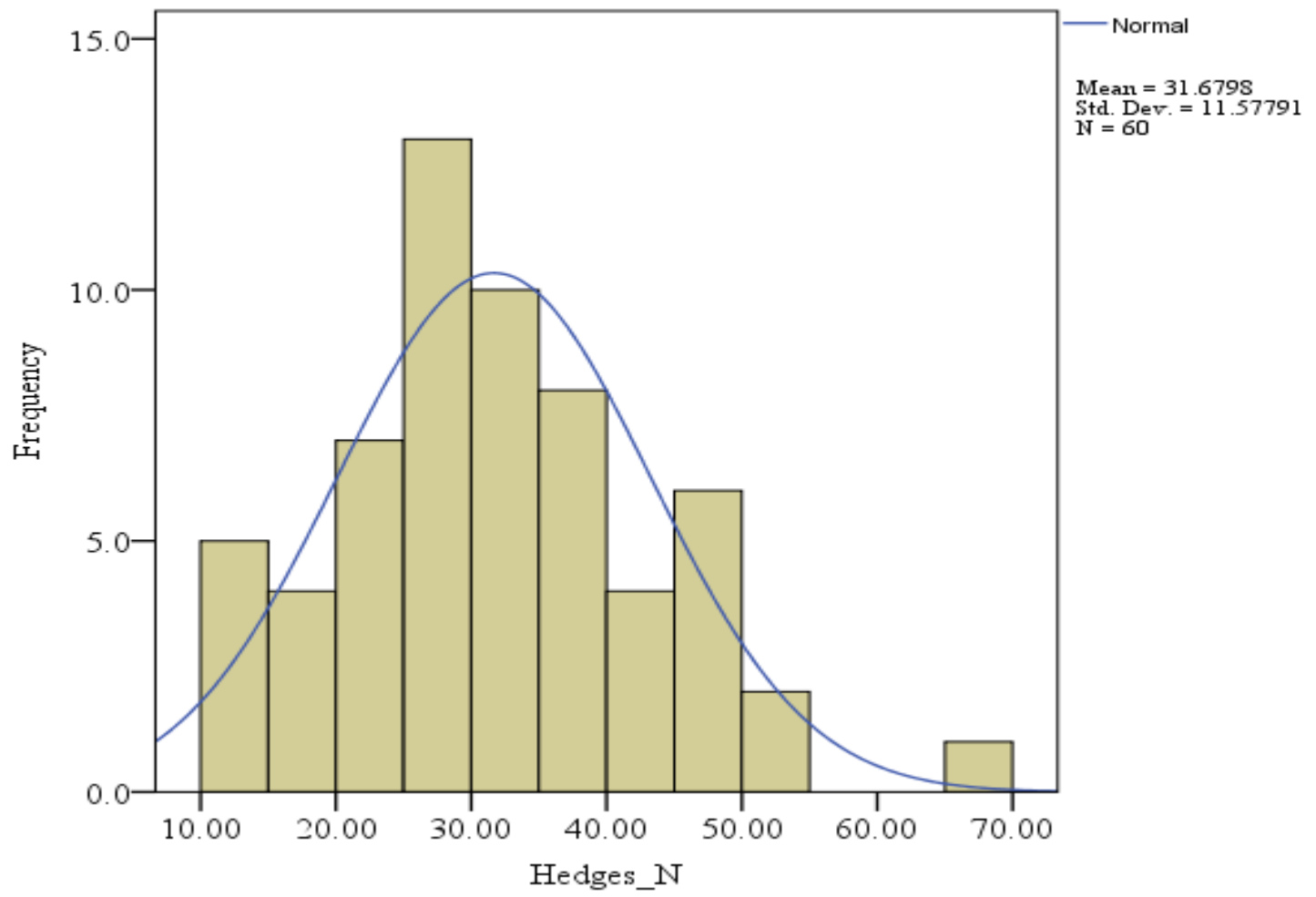

Figure B6. The histogram of frequency distribution of total hedges use.

\section{Appendix C: The Results of Mann-Whitney Tests}

Table C1

The Difference Between Chinese Students and American Students in Model Auxiliary Type of Hedges Use

\begin{tabular}{lllll}
\hline Ranks & & & & \\
\hline & First language & N & Mean rank & Sum of ranks \\
\hline \multirow{2}{*}{ Aux_N } & Chinese & 30 & 28.27 & 848.00 \\
& English & 30 & 32.73 & 982.00 \\
& Total & 60 & & \\
\hline
\end{tabular}

Test Statistics

\begin{tabular}{ll}
\hline & Aux_N \\
Mann-Whitney U & 383.000 \\
Wilcoxon W & 848.000 \\
Z & -.991 \\
Asymp. Sig. (2-tailed) & .322 \\
\hline
\end{tabular}

Grouping Variable: First language

Table C2

The Difference Between Chinese Students and American Students in Lexical Verb Type of Hedges Use

\begin{tabular}{llllc}
\hline Ranks & & & \\
\hline \multirow{3}{*}{ Verb_N } & First language & N & Mean rank & Sum of ranks \\
& Chinese & 30 & 25.57 & 767.00 \\
& English & 30 & 35.43 & 1063.00 \\
\hline
\end{tabular}




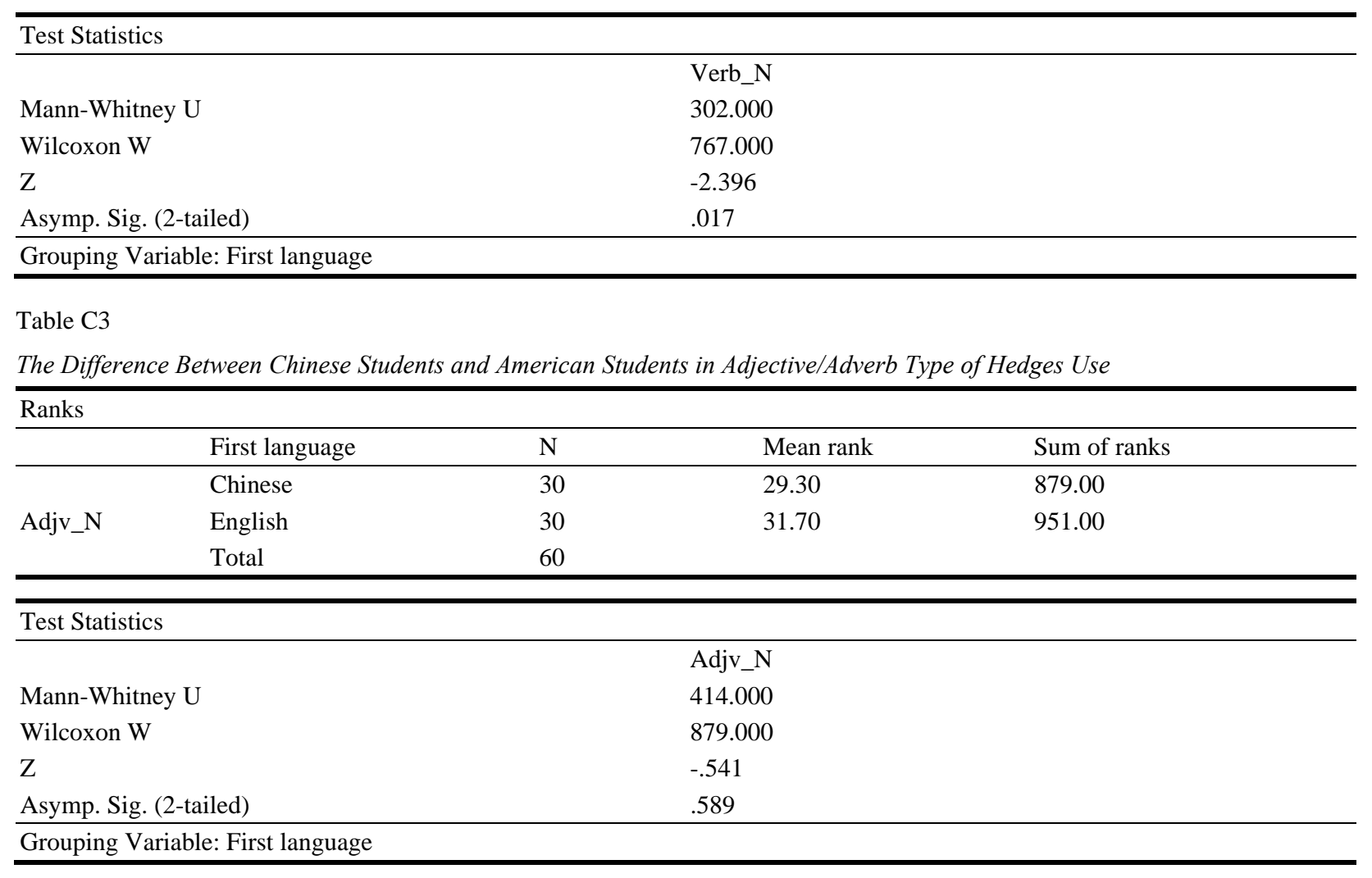

Table C4

The Difference Between Chinese Students and American Students in Phrase Type of Hedges Use

\begin{tabular}{|c|c|c|c|c|}
\hline \multicolumn{5}{|l|}{ Ranks } \\
\hline & First language & $\mathrm{N}$ & Mean rank & Sum of ranks \\
\hline \multirow{3}{*}{ Phrase_N } & Chinese & 30 & 32.60 & 978.00 \\
\hline & English & 30 & 28.40 & 852.00 \\
\hline & Total & 60 & & \\
\hline \multicolumn{5}{|c|}{ Test Statistics } \\
\hline \multicolumn{5}{|c|}{ Phrase_N } \\
\hline \multicolumn{3}{|c|}{ Mann-Whitney U } & \multicolumn{2}{|c|}{387.000} \\
\hline \multicolumn{2}{|c|}{ Wilcoxon W } & & \multicolumn{2}{|c|}{852.000} \\
\hline \multicolumn{2}{|c|}{$\mathrm{Z}$} & & \multicolumn{2}{|c|}{-1.576} \\
\hline \multicolumn{2}{|c|}{ Asymp. Sig. (2-tailed) } & & \multicolumn{2}{|c|}{.115} \\
\hline \multicolumn{5}{|c|}{ Grouping Variable: First language } \\
\hline
\end{tabular}

Table C5

The Difference Between Chinese Students and American Students in Sentence Structure Type of Hedges Use

\begin{tabular}{lllll}
\hline Ranks & & & & \\
\hline & First language & N & Mean rank & Sum of ranks \\
\hline \multirow{3}{*}{ Sentence_N } & Chinese & 30 & 30.70 & 921.00 \\
& English & 30 & 30.30 & 909.00 \\
& Total & 60 & & \\
\hline
\end{tabular}




\begin{tabular}{|c|c|c|c|c|}
\hline \multicolumn{5}{|l|}{ Test Statistics } \\
\hline \multicolumn{5}{|c|}{ Sentence_N } \\
\hline \multicolumn{3}{|l|}{ Mann-Whitney U } & \multicolumn{2}{|l|}{444.000} \\
\hline \multicolumn{3}{|l|}{ Wilcoxon W } & \multicolumn{2}{|l|}{909.000} \\
\hline \multicolumn{3}{|l|}{$\mathrm{Z}$} & \multicolumn{2}{|l|}{-.089} \\
\hline \multicolumn{3}{|l|}{ Asymp. Sig. (2-tailed) } & \multicolumn{2}{|l|}{.929} \\
\hline \multicolumn{5}{|c|}{ Grouping Variable: First language } \\
\hline \multicolumn{5}{|l|}{ Table C6 } \\
\hline \multicolumn{5}{|c|}{ The Difference Between Chinese Students and American Students in Total Hedges Use } \\
\hline \multicolumn{5}{|c|}{ Ranks } \\
\hline & First langauge & $\mathrm{N}$ & Mean rank & Sum of ranks \\
\hline \multirow{3}{*}{ Hedges_N } & Chinese & 30 & 29.50 & 885.00 \\
\hline & English & 30 & 31.50 & 945.00 \\
\hline & Total & 60 & & \\
\hline \multicolumn{5}{|l|}{ Test Statistics } \\
\hline \multicolumn{5}{|c|}{ Hedges_N } \\
\hline \multicolumn{3}{|l|}{ Mann-Whitney U } & \multicolumn{2}{|l|}{420.000} \\
\hline \multicolumn{2}{|l|}{ Wilcoxon W } & & \multicolumn{2}{|l|}{885.000} \\
\hline \multicolumn{2}{|l|}{$\mathrm{Z}$} & & \multicolumn{2}{|l|}{-.444} \\
\hline \multicolumn{3}{|c|}{ Asymp. Sig. (2-tailed) } & \multicolumn{2}{|l|}{.657} \\
\hline \multicolumn{5}{|c|}{ Grouping Variable: First language } \\
\hline
\end{tabular}

